\title{
Starch-based diet, plant-based diet or fasting may be beneficial to individuals suffering from sequelae of SARS-CoV-2 infection
}

\author{
Yuhan Lin ${ }^{1}$, Lipeng Liao ${ }^{1}$, Chanyuk Lam ${ }^{1}$, Jess Lan Ouyang ${ }^{1}$, Min Fu $^{2}$, Man Tang ${ }^{1}$, Qiuyun Liu ${ }^{1, *}$ \\ ${ }^{1}$ School of Life Sciences, Sun Yat-Sen University, Guangzhou 510275, China. \\ ${ }^{2}$ Deyang Health Committee, Sichuan, China
}

*Correspondence author: Qiuyun Liu, School of Life Sciences, Sun Yat-sen University, Guangzhou 510275, China.

E-mail address: 1sslqy@ mail.sysu.edu.cn (Q. Liu) 


\begin{abstract}
High valine plus glycine content is a hallmark of the proteins in SARS-CoV-2 and SARS viruses, and it triggers the formation of aggregates between the two amino acid residues and calcium oxalate. As a result, SARS-CoV-2 infected individuals suffer from breathing difficulties. Human proteins with high valine plus glycine percentages can be induced during infection and these proteins may be partially retained in the body post-infection, giving rise to sequelae after disease recovery. To ameliorate such problems, starch-based diet, plant-based diet, or intermittent fasting with boiled rice water or fruit juice may be favorable to patients recovered from SARS-CoV-2 infections as they limit the intake of essential amino acids or all amino acids. Lysine supplement can be beneficial as lysine rich proteins attract chloride and solubilize insoluble and stiff calcium oxalate. However, clinical trials must be conducted to fully justify the use of lysine.
\end{abstract}

Keywords

High valine plus glycine content; SARS-CoV-2; aggregates; calcium oxalate 
Despite that lysine supplement is perhaps favorable for heart disease patients as previously reported or recovered patients with constipation symptoms, it increases tumor risks to cancer susceptible human subpopulation with diarrhea since it enhances the buildup of $\mathrm{Cl}^{-}$and mutagenic and carcinogenic $\mathrm{HCl}$ (1418). The side chain of arginine may have affinity to divalent cations, and hence enhance the formation of calcium oxalate. The presence of numerous valine and glycine residues in viral proteins can be the cause of thrombosis during vaccination.

\section{Conflict of interest}

None declared.

\section{ACKNOWLEDGMENT}

This work was supported by the Science and Technology Transformation Program of Sun Yat-sen University of China (33000-18843234) and Guangzhou Science and Technology Program (201804010328) to Q. Liu. We thank Yan Shi for editing.

\section{REFERENCES}

1. Wan Y, Yan S, Zhang Y, An S, Yang K, Xu H, Gan T, et al. The Pneumonia outbreak: High isoleucine and high valine plus glycine contents are features of the proteins of COVID-19 virus. Preprints. 2020. doi: 10.20944/preprints202002.0289.v5

2. Yan S, Zhang Y, Liu Q. Why COVID-19 virus is so deadly to cancer patients? Eur J Cancer Prev. 2020;29(4):365.

3. Lam C, Lin Y, Liao L, Tang M, Fu M, Liu Q. Simultaneous fasting may contain SARS-CoV-2 infection. OSF Preprints. 2021. osf.io/zn9rg. https://osf.io/zn9rg

4. Liao L, Gan T, Lin Y, Lam C, Ouyang JL, Liu Q. The need for accessibility to chloride to solubilize insoluble salts may underlie some cases of hypertension. OSF Preprints. 2021. osf.io/z2m48. https://osf.io/z2m48

5. Liao L, Lin Y, Lam C, Ouyang JL, Fu M, Tang M, \& Liu Q. The requisite for acid generation to solubilize insoluble salts may account for surge of glucose levels in some diabetes patients. OSF Preprints. 2021. osf.io/fvxy6. https://osf.io/fvxy6

6. Lin Y, Liao L, Zhang Y, Lam C, Tang M, Liu Q. Why dexamethasone can reduce mortality rate among patients with viral infections. OSF Preprints. 2021. osf.io/jrpg2. https://osf.io/jrpg2

7. An S, Zhang X, Shi Y, Zhang J, Wan Y, Wang Y, et al. High glycine content in TDP-43: a potential culprit in limbic-predominant age-related TDP-43 encephalopathy. J Inter Med Res. 2020;48(6):300060520929853. https://doi.org/10.1177/0300060520929853

8. Qi J, Zhang X, Chen Z, Shi Y, Liu Q. Transgenic proteins rich in valine or glycine are concerns for heart disease patients. Eur J Prev Cardiol. 2018;25(8):883-4. https://doi.org/10.1177/2047487318758111

9. Wan Y, Zhang J, Li X, Wang Y, Liu Q. Cellular states and secondary chemical bonding: a biochemical view of major human diseases. Biochem Insights. 2019;12:1178626419877846. https://doi.org/10.1177/1178626419877846 
10. Wang Y, Tang JW, Ma WQ, Feng J, Feng J. Dietary zinc glycine chelate on growth performance, tissue mineral concentrations, and serum enzyme activity in weanling piglets. Biol Trace Elem Res 2010;133(3):325-34. https://doi.org/10.1007/s12011-009-8437-3

11. Zhang X, Ma X, Gan T, Shi Y, Wang Y, Liu Q. Secondary Chemical Bonding between Insoluble Calcium Oxalate and Carbonyl Oxygen Atoms of GLY and VAL Residues Triggers the Formation of A $\beta$ Aggregates and Their Deposition in the Brain. ACS Chem Neurosci. 2020;11(24):400711.https://doi.org/10.1021/acschemneuro.0c00662

12. Zhou Y, Shi Y, Wan Y, Ye ZW, Liu Q. Why the Mediterranean diet lowers the risk of heart disease. Eur J Prev Cardiol. 2017;24:1788-9. doi:10.1177/2047487317730653

13. Gan T, Fu M, Wu J, Wen L, Liu Q. How to design carbohydrate diet regimens for heart disease patients. Eur J Prev Cardiol. 2018; 25(9): 979-80. https://doi.org/10.1177/2047487318766602

14. An S, Li X, Tang M, et al. The role of acetate in the antagonization of oxalate - A potential causative molecule for heart disease and cancer death. Nat Prod Commun. 2020;15(3):1-4.

15. Tang M, Zhou Y, Li Y, et al. Hydrogen donors and acceptors and basic amino acids jointly contribute to carcinogenesis. Med Hypotheses. 2017;98:42-44.

16. Shi Y, An S, Wan Y, et al. How to best use acetic acid for the prevention of heart disease and cancer. Eur J Prev Cardiol 2019; 26:437-8.

17. Wan Y, Li J, Liu Q. Vinegar production and cancer risk. Eur J Cancer Prev. 2019;28(4):382.

18. Wan Y, Ma X, Li Y, Huang X, Gan T, Xu L, Cao W, Liu Q. Local strong acids: A driving force for metastasis. Med Hypotheses.2020; 144: 110221. 